\title{
ORIGIN OF COMETS
}

"T HE Distribution of the Directions of Perihelia of Long-Period Comets" is the title of a paper by J. G. Tyror in Mon. Not. Roy. Astro. Soc. (117, 4 ; 1957), in which he deals specially with Lyttleton's theory of the origin of comets (see ibid., 103, 465; 1948 ; and "The Comets and their Origin", Cambridge, 1953). This theory envisaged a process of accretion of dust particles by the Sun as it passed through interstellar dust clouds, and while it explained some of the observed features of comets and of their orbits, like many other theories it presented certain difficulties. If the theory is accepted, then comets must originally have come from preferred positions in the sky, and although the diffusion of the perihelion points of such a family would probably occur, it is considered that some traces of perihelion groupings, at lesst for families born during recent cloud passages, could be detected.

Mr. Tyror examines the distribution of perihelion points of long-period comets with the object of determining whether there is any evidence of such grouping. Previous investigations by W. H. Pickering and others suggested that there was some evidence of the grouping of aphelion points, but it has been considered advisable to revise these results in the light of additional information which has appeared since the completion of their work. The present survey does not include short-period comets and periodic comets because planetary perturbations would have eradicated reliable evidence of any original grouping; while it is true that all orbits would have been affected to some extent, the investigation has been carried out only for comets suffering slight perturbations. The whole subject is beset with difficulties because it is inevitable that available data must suffer to some extent from observational selection. A detriled description of the contents of the paper is quite impossible in a short note, and it will suffice to deal with the data utilized and the conclusions that have been reached.

Investigations of the positions of 448 comets with periods greater than 200 years were made, and of these 67 had calculated eccentricities greater than 1 , 263 equal to 1 , and 118 less than 1 . There is evidence that the perihelion points of long-period comets tend to be distributed non-randomly, and it is pointed out that this tendency may be due to observational selection or to a real grouping phenonenon, or even to a combination of both. The first of these has undoubtedly played a prominent part in the discovery of comets, and other vitiating factors in the analysis are mentioned. The conclusions are summarized at the end of the paper and are briefly as follows.

The departure from randomness in the observed distribution of porihelion points of the comets considered is strongly suggested, and there is evidence indicating a preference for the perihelion points to lie near a particular great plane. In addition, the centre of the observed distribution appears to be significantly displaced towards a point on the celestial sphere. The identification of the plane with the plane of the Galexy and of the point as that lying in a direction opposed to that of the Sun's motion cannot be considered unreasonable. These suggestions may be interpreted in the light of Lyttleton's accretion hypothesis, according to which the perihelion positions of comets should show a tendency to lie near the apex of the Sun's motion relative to the local interstellar cloud, and further, the distribution of perihelion points would have a preference for lying near the galactic plane.

\section{QUALITY IN MEAT}

TOW that the supply of meat is approaching the 1 level of demand, quality is again assuming economic importance. Definitions and tests of quality have been examined by D. M. Joubert of the Department of Animal Husbandry, University of Pretoria, who has also examined the economic consequences which follow from changed attitudes towards quality (Colonial Plant and Animal Products, 6 , No. 2 ; 1956).

Only a few decades ago quality in beef meant wellmarbled meat covered by a thick layer of fat. Not only did the average consumer during those times demand more fat owing to the bodily needs imposed by hard manual labour, but also excess fat was used for other purposes, chiefly cooking. At present, cooking fats can be obtained from cheaper sources, the consumers perform less manual labour and are better protected in their homes against cold conditions, and consequently demand the minimum of fat with their beef. Since butchers are concentrating on carcasses of young animals, it is not unlikely that the optimum carcass weight will become even lower in future.

When considering quality in the case of mutton and lamb, the size factor is of primary importance. With the size of the average family decreasing all over the world and with cooking facilities less specious than they used to be, the general demand is for small joints. Preference is given to a whole joint of small size over half a large one since the latter, because of its cut surface, does not retain its succulence so well and leads to greater wastage through exudation. As in beef, a minimum of fat is preferred. With the rapid development of the dairy industry in recent years and with progress in the manufacture of margarine, the excess fat is no longer widely used as a substitute for butter and goes to weste.

Definition of quality in mutton is particularly complicated because of the differences in the taste of individuals in respect of flavour. Mutton produced from early-maturing sheep (as, for example, Southdown, Suffolk) raised and fattened in stalls may appear extremely attractive and, therefore, be labelled as of high quality. On the other hand, mutton produced from late-maturing shoep breods (such as Scotch Blackface, and even possibly Merino) off extensive pastures may, by comparison, appear unattractive and may be labelled as being of low quality.

The quality of pork is determined primarily by the amount and nature of the subcutaneous back fat. The amount of fat preferred with pork by consumers in Britain at present is considerably less than a few decades ago, while in South Africa, for example, a large amount of fat has never been tolerated except 
where it was required for purposes other than eating. It was not realized that lean pork can be produced provided the right type of animal is used and that correct feeding is practised, and the consumption of pork in South Africa remained extremely low until quite recently. On the contrary, in certain Continental countries the general taste demands rather more fat with pork, and a certain amount of excess fat is required by butchers for the production of a great many varieties of sausage and salami. Consequently the type of pig raised in the central European States (such as Germany and Hungary) differs markedly in its conformation from that which is considered as the ideal type in Great Britein and South Africa.

The final factors determining the quality of a piece of meat, and one which often nullifies all factors considered prior to its cooking, centre around the purely human element, namely, the ability of the cook-either to spoil the good qualities of meat through inefficiency, or to improve to such an extent the quality of a poor sample of meat through skill and knowledge of cooking, per se, that its pre-cooked quality remains unquestioned.

\title{
ASSAY OF VITAMIN A OILS
}

\author{
By Prof. E. BRUNIUS \\ National Institute of Public Health, Tomteboda, Sweden
}

N 1954 a Vitamin Assay Commission was set up by the Food Division of the Applied Chemistry Section of the International Union of Pure and Applied Chemistry.

The official members of the Vitamin Assay Com. mission are: Prof. E. Brunius (Sweden, chaiman), Dr. W. F. J. Cuthbertson (Great Britain), Dr. M. Kofler (Switzerland), Dr. B. L. Oser (United States) and Prof. H. Simonnet (France). The Commission has held four Conferences : in London 1954, Zurich 1955, Amsterdam 1956, and Paris 1957. In all the Conferences, besides members of the commission, the following participated : Dr. D. W. Kent-Jones (Great Britain, honorary secretary of the Food Division), Prof. R. Nicolaysen (Norway, member of the Food Division), Mr. E. Hayes (Great Britain), and Mr. R. J. Taylor (Great Britain). In addition the following were present at the Amsterdam conference : Dr. J. Straub (The Netherlands, member of the Food Division), and Dr. C. Engel (The Netherlands) ; and at the Paris Conference : Dr. K. Dürrenmatt (Switzerland, chairman of the Food Division), Prof. J. Brüggemann (Germany), Dr. C. Engel (The Netherlands), Dr. A. François (France), and Dr. J. Tiews (Germany).

One of the Commission's tasks was to develop a standardized method for the assay of vitamin A oils which would be accepteble in internationsl trade.

Current vitamin A assay methods are based on the recommendations made by the Subcommittee on Fat-Soluble Vitamins of the World Health Organization. In its report ${ }^{1}$ the International Unit of vitamin A potency is defined in terms of the activity of crystal. line all-trans vitamin A acetate, and the conditions for the spectrophotometric assay of the vitamin are broadly set down. The elaboration of methods in different countries, however, has led to divergences in the assessment of vitamin A potency, and this has given rise to difficulties in the international trade in vitamin A oils. It was therefore considered an urgent task for the Commission to attempt to develop a standardized method of assay.

Vitamin A oils vary widely in quality and potency. At the outset the Commission decided, on the evidence available, to consider them in two categories accord. ing as their spectrophotometric characteristics approached those of pure vitamin $A$ or not. For oils in the first category (comprising most fish-liver oils and vitamin A concentrates) a correction procedure based on the Morton-Stubbs principle ${ }^{2}$ was considered sufficient. For oils in the second category (comprising whale-liver oils and other oils having a high proportion of irrelevantly light-absorbing materials) a chromatographic step should precede the determination of extinction and the application of correction. All measurements should be made on the unsaponifiable matter of the oils. It was suggested that this should be isolated by the method described in the United States Pharmacopeia.

The Commission organized three collaborative experiments, in which laboratories in Europe, Japan and the United States co-operated. Thirty-seven laboratories participated in the first experiment, thirty-four in the second and nine in the third.

The main purposes of the experiments were : (1) to ascertain the loss of vitamin $A$ in isolating unsaponifiable matter of oils according to the method of the United States Pharmacopeia; (2) to investigate whether isopropanol and absolute ethanol could be used as alternative solvents in messuring extinotion; (3) to determine the constants for a correction formula based on the Morton-Stubbs principle ${ }^{2}$; (4) to ascertain the loss of vitamin $A$ in a chromatographic method suggested for samples in the second category; (5) to investigate the selectivity of this method; (6) to compere two techniques for following the movement of vitamin A through the chromatographic column; and (7) to estimate the experimental errors in the vitamin $A$ potencies of oils in both categories as assayed by the procedure suggested.

A vitamin A reference standard containing alltrans vitamin A acetate in vegetable oil (nominal potency 100,000 I.U. per gm.) and samples of oils belonging in the second category were used as test materials.

In the chromatography, the unsaponifiable matter of the sample was passed through a column of weakened alumina and the vitamin was eluted with mixtures of light petroleum and ether. The movement of the vitamin was followed by testing aliquots of the eluate with antimony trichloride solution and, in the third experiment, alternatively by observing the column in weak ultra-violet light. Two different samples of alumina were used by most participants.

A statistical screening procedure, based on Chauvenet's criterion ${ }^{3}$, was applied to the results.

The main outcome of the experiments can be summarized as follows. The average loss of vitamin A in isolating the unsaponifiable matter of the vitamin A reference standard was found to be 1.2 per cent in 